\title{
Clinical implications of differentiating between types of post- tracheostomy tracheal stenosis
}

\author{
Beomsu Shin $^{1 *}$, Kang Kim ${ }^{1 *}$, Byeong-Ho Jeong ${ }^{1}$, Jung Seop Eom ${ }^{2}$, Won Jun Song ${ }^{3}$, Hojoong Kim ${ }^{1}$ \\ ${ }^{1}$ Division of Pulmonary and Critical Care Medicine, Department of Medicine, Samsung Medical Center, Sungkyunkwan University School of \\ Medicine, Seoul, South Korea; ${ }^{2}$ Department of Internal Medicine, Pusan National University School of Medicine, Busan, South Korea; ${ }^{3}$ Department \\ of Critical Care, Kangbuk Samsung Hospital, Seoul, South Korea \\ Contributions: (I) Conception and design: BH Jeong, H Kim; (II) Administrative support: JS Eom, WJ Song, H Kim; (III) Provision of study materials \\ or patients: BH Jeong, H Kim; (IV) Collection and assembly of data: B Shin, K Kim, JS Eom, WJ Song; (V) Data analysis and interpretation: B Shin, \\ K Kim, BH Jeong, H Kim; (VI) Manuscript writing: All authors; (VII) Final approval of manuscript: All authors. \\ *These authors contributed equally to this work. \\ Correspondence to: Byeong-Ho Jeong, MD, PhD. Division of Pulmonary and Critical Care Medicine, Department of Medicine, Samsung Medical \\ Center, Sungkyunkwan University School of Medicine, 81 Irwon-ro, Gangnam-gu, Seoul 06351, South Korea. Email: myacousticlung@gmail.com.
}

\begin{abstract}
Background: Post-tracheostomy tracheal stenosis (PTTS) can be divided into four types according to stenosis mechanism and site: subglottic, stoma, cuff, and tip granuloma. However, there is little information available regarding clinical differences among types of PTTS; therefore, we evaluated the clinical differences between these types.
\end{abstract}

Methods: We retrospectively evaluated 99 PTTS patients who underwent interventional bronchoscopy between 2004 and 2014. Patients were divided into two groups according to pathophysiological similarities as follows: subglottic or stoma type $(n=59)$ and cuff or tip type $(n=40)$.

Results: There were no differences in baseline characteristics between groups. However, silicone stents were more frequently needed in patients with subglottic or stoma type stenosis $(76 \%)$ than those with cuff or tip type stenosis $(55 \%, \mathrm{P}=0.031)$ to maintain airway patency. On the contrary, permanent tracheostomy was more frequently performed in patients with cuff or tip type stenosis (50\%) than those with subglottic or stoma type stenosis $(19 \%, \mathrm{P}=0.002)$. Finally, successful removal of the tracheostomy tube without surgery and procedure- or disease-related mortality were more frequently achieved in patients with subglottic or stoma type stenosis $(71 \%)$ than those with cuff or tip type stenosis $(45 \%, \mathrm{P}=0.012)$.

Conclusions: Although there were no significant differences in baseline characteristics between PTTS types, patients with subglottic or stoma type stenosis had more favorable outcomes than those with cuff or tip type stenosis. Therefore, it could be important to distinguish between types of PTTS when assessing prognosis.

Keywords: Post-tracheostomy tracheal stenosis (PTTS); interventional bronchoscopy; airway stent; treatment outcome

Submitted May 29, 2017. Accepted for publication Oct 17, 2017.

doi: $10.21037 /$ jtd.2017.10.99

View this article at: http://dx.doi.org/10.21037/jtd.2017.10.99

\section{Introduction}

Tracheal stenosis following tracheostomy is a wellestablished type of acquired benign stenosis in critically ill patients $(1,2)$. Post-tracheostomy tracheal stenosis
(PTTS) occurs in approximately $1-2 \%$ of patients as a late complication of tracheostomy $(3,4)$. Although tracheal resection and end-to-end anastomosis is the treatment of choice for this type of lesion, postoperative complications were significantly increased in association with poor general 

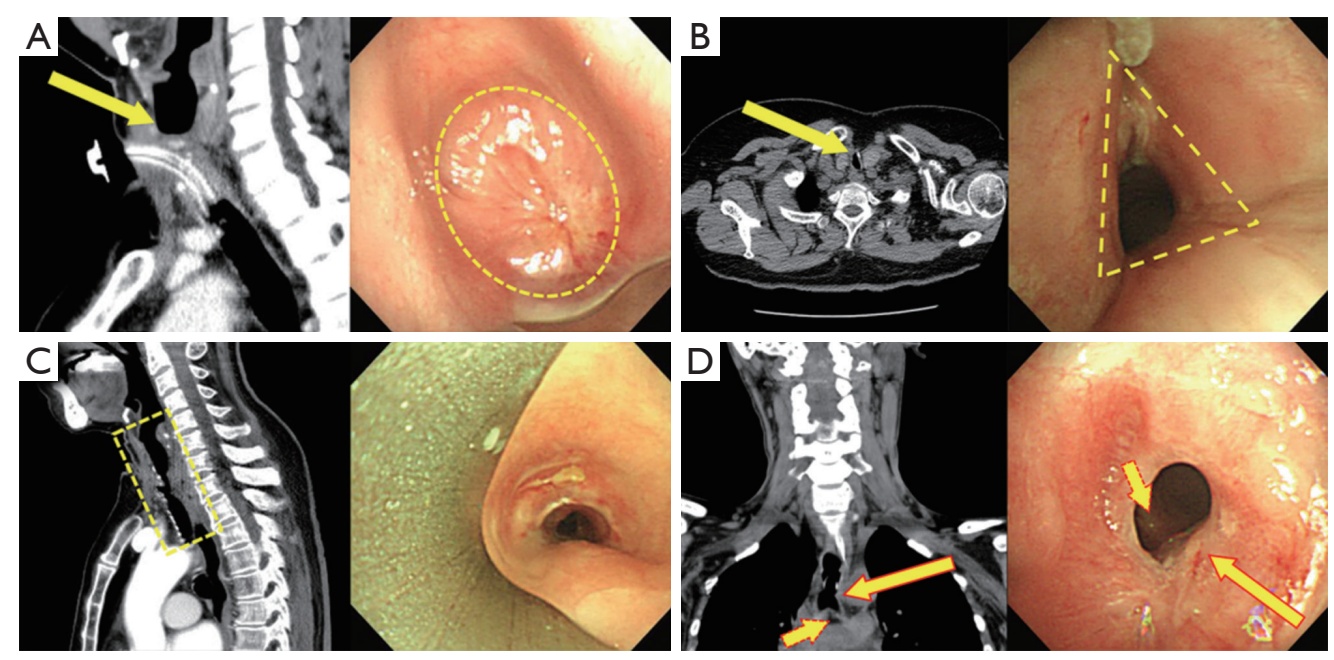

Figure $1 \mathrm{CT}$ and bronchoscopic findings according to PTTS type. (A) Subglottic type. Subglottic area was completely obstructed by fibrotic stricture (arrow and circle); (B) stoma type. Upper trachea at the tracheostomy stoma was narrowed by cartilage fracture and fibrosis (arrow). Tracheal stenosis of triangular shape was observed during the bronchoscopy (dotted triangle); (C) cuff type. Mid-trachea at the cuff level was narrowed by fibrotic bands (dotted square); (D) tip granuloma type. Distal trachea just above the carina was narrowed by fibrosis (long solid arrow: fibrotic band; short dotted arrow: carina). CT, computed tomography; PTTS, post-tracheostomy tracheal stenosis.

health and comorbidities (5,6). Interventional bronchoscopy is an acceptable alternative to surgery for many inoperable patients, considering the high comorbidities of patients with PTTS $(7,8)$.

PTTS can be subdivided into four types in accordance with stenosis site and pathophysiology as follows: subglottic, stoma, cuff, and tip types (9). PTTS occurs at subglottic or stomal sites, caused by inadequate incisions during tracheostomy procedures. In addition, PTTS sometimes occurs at the cuff or tip of the tracheostomy tube, caused by inadequate management of problems such as high cuff pressure and excessive irritation by the suction tip.

Each type of PTTS may have different clinical characteristics and optimal management may require different practices. However, there have been few studies comparing types of PTTS. Therefore, we investigated the clinical characteristics of patients with each type of PTTS who underwent interventional bronchoscopy.

\section{Methods}

\section{Patients}

We reviewed all 1,064 consecutive patients with airway stenosis who underwent interventional bronchoscopy at Samsung Medical Center (a 1,979-bed, universityaffiliated, tertiary care referral hospital in Seoul, South
Korea) between January 2004 and December 2014. Of these patients, 99 were identified to have tracheal stenosis after tracheostomy. Some patients who were reported in a previous study were re-evaluated in terms of clinical outcomes for extension of follow-up duration (10). Patients with PTTS were classified into two groups, "subglottic or stoma" and "cuff or tip," based on clinical, bronchoscopic, and radiological findings, which were thoroughly reviewed by three of the authors (B Shin, K Kim, and BH Jeong). Each type is detailed in Figure 1.

This retrospective observational study received Institutional Review Board of Samsung Medical Center approval (IRB No. 2016-08-139). The need for informed consent was waived because patient information was anonymized and de-identified prior to analysis.

\section{Airway intervention techniques}

We assessed airway anatomy and function using chest radiography, computed tomography (CT), flexible bronchoscopy, and, if possible, pulmonary function tests (PFT). When tracheal stenosis was greater than $50 \%$ in cross-sectional area and/or the patient had symptomatic dyspnea limiting activities of daily living, interventional bronchoscopy was performed (11-13).

Interventional bronchoscopy was performed according 
to standard techniques after induction of general anesthesia and intubation with a rigid bronchoscope tube (Bryan Co., Woburn, MA, USA) $(14,15)$. Depending on the subtype of tracheal stenosis and the general health status of the patient, individualized intervention techniques were designed, such as mechanical dilation, balloon dilation, laser therapy, and insertion of a silicone stent, as previously reported (10,16-18). When localized dense fibrosis was observed, a laser was used to cut the fibrotic band. When mechanical dilation or laser treatment did not satisfactorily preserve airway patency, airway stents were implanted using the standard technique described by Dumon (19). A stent of the appropriate size was folded longitudinally, introduced into a stent pusher (Bryan Corp., Woburn, MA, USA), and properly repositioned using alligator forceps. If the stent did not fully expand, balloon dilation was performed (20).

Patients could usually return to normal activity 1 to 3 days after the procedure and underwent simple chest radiography and spirometry at $1,3,6,9$, and 12 months after procedures to evaluate each patient's condition. Bronchoscopy and chest CT were performed when needed to reassess stent location and airway patency. Stent removal was planned when the patients had exhibited stable conditions for 1 year and when air pockets were confirmed on CT. An air pocket was defined as tracheobronchial air columns in the space between the outer surface of the stent and the adjacent airway wall (21). If restenosis or malacia developed, stent insertion was repeated. If the procedures were ineffective, surgical resection and anastomosis or permanent tracheostomy was considered.

\section{Stents}

The types of airway silicone stents used during the study period included the Natural stent (M1S Co., Seoul, Korea), the DUMON stent (Novatech, La Ciotat, France), and the Montgomery T-tube (Koken, Tokyo, Japan). The Natural stent is a silicone stent developed at the Samsung Medical Center in 2002. Studies in a canine model of tracheal stenosis and clinical studies in patients with benign tracheobronchial stenosis have shown that the Natural stent is as effective and safe as the Dumon stent $(22,23)$. However, the production of Natural stents ceased due to commercial issues. Dumon stents have been commercially available for medical use since 2015. Montgomery T-tubes were used in patients with high probability of mucostasis or with tracheal stenosis close to the glottis $(24,25)$. External fixation with unabsorbable nylon was performed in patients treated with DUMON or Natural stents.

\section{Data collection}

Demographic data, stenosis characteristics, and clinical outcomes were obtained as in our previous study (10). In short, poor performance was defined as an Eastern Cooperative Oncology Group (ECOG) grade $\geq 3$, which means the patient is confined to bed or a chair more than $50 \%$ of their waking hours (26). The degree of tracheal stenosis was determined according to the Myer-Cotton stenosis grading system (27). In this study, respiratory failure requiring intubation or emergent tracheostomy before interventional bronchoscopy was considered grade IV. Final success was assessed by two methods: (I) the "successful removal of tracheostomy tube" group included patients who maintained airway patency without disease-related or procedure-related mortality or surgical management and could have the tracheostomy tube removed, and (II) the "successful removal of airway prosthesis" group included patients whose airway patency was maintained without an airway stent.

\section{Statistical analyses}

All results are presented as median and interquartile range (IQR) or numbers (percentages). Continuous variables are compared using the Mann-Whitney U test. Categorical variables are compared using Pearson's chi-square test or Fisher's exact test. Forced expiratory volume in one second $\left(\mathrm{FEV}_{1}\right)$ before and after the procedure was compared using the Wilcoxon signed rank test. To identify risk factors independently associated with clinical outcomes, we conducted multivariate analyses using logistic regression models with backward selection. Statistical differences were considered significant at $\mathrm{P}<0.05$. All analyses were performed using SPSS software (IBM SPSS Statistics ver. 22, Chicago, IL, USA).

\section{Results}

\section{Baseline characteristics}

Of the total 99 patients with PTTS, the median patient age was 58 years, $52.5 \%$ of patients were male, and $62.6 \%$ of patients had poor performance status (Table 1 and Table S1). There were no statistically significant differences in demographic data between groups except for body mass index (BMI). BMI was lower in patients with cuff or tip 
Table 1 Baseline characteristics

\begin{tabular}{|c|c|c|c|c|}
\hline Variables & Total $(n=99)$ & Subglottic or stoma type $(n=59)$ & Cuff or tip type $(n=40)$ & $P$ value \\
\hline Male & $52(52.5)$ & $33(55.9)$ & $19(47.5)$ & 0.421 \\
\hline Body mass index $\left(\mathrm{kg} / \mathrm{m}^{2}\right)$ & $21.4(19.2-23.2)$ & $21.7(20.0-23.6)$ & $20.7(17.8-22.4)$ & 0.042 \\
\hline Poor performance status ${ }^{*}$ & $62(62.6)$ & $34(57.6)$ & $28(70.0)$ & 0.290 \\
\hline Medical & $72(72.7)$ & $46(78.0)$ & $26(65.0)$ & 0.174 \\
\hline Neurological disease & $35(35.3)$ & $19(32.2)$ & $16(40.0)$ & 0.521 \\
\hline Respiratory failure & $19(19.2)$ & $14(23.7)$ & $5(12.5)$ & 0.200 \\
\hline Heart failure & $9(9.1)$ & $6(10.2)$ & $3(7.5)$ & 0.736 \\
\hline Drug intoxication & $2(2.0)$ & $1(1.7)$ & $1(2.5)$ & 1.000 \\
\hline Obesity & $1(1.0)$ & $1(1.7)$ & 0 & 1.000 \\
\hline Trauma & $27(27.3)$ & $13(22.0)$ & $14(35.0)$ & 0.174 \\
\hline Previous treatment history at another hospital & $26(26.3)^{\dagger}$ & $13(22.0)$ & $13(32.5)$ & 0.256 \\
\hline $\begin{array}{l}\text { Severity of tracheal stenosis (Myer and } \\
\text { Cotton grade }{ }^{\ddagger} \text { ) }\end{array}$ & & & & 0.660 \\
\hline I & $5(5.0)$ & $3(5.1)$ & $2(5.0)$ & \\
\hline II & $10(10.1)$ & $5(8.5)$ & $5(12.5)$ & \\
\hline Granulation & $56(56.6)$ & $33(55.9)$ & $23(57.5)$ & 1.000 \\
\hline Malacia & 20 (20.2) & $11(18.6)$ & $9(22.5)$ & 0.799 \\
\hline Mixed & $48(48.5)$ & $32(54.2)$ & $16(40.0)$ & 0.219 \\
\hline
\end{tabular}

Data are presented as $n(\%)$ or median (IQR). *, Eastern Cooperative Oncology Group (ECOG) performance grade $\geq 3$ means patients were confined to a bed or chair more than $50 \%$ of the day; ${ }^{\ddagger}$, categorization based on the percentage of reduction in cross-sectional area: grade 1, $\leq 50 \%$ lumenal stenosis; grade II, 51-70\% lumenal stenosis; grade III, 71-99\% lumenal stenosis; grade IV, no lumen, intubation or tracheostomy state due to respiratory failure before interventional bronchoscopy; ${ }^{\dagger}$, in patients with subglottic or stoma type, Montgomery T-tube $(n=5)$, surgical correction $(n=4)$, ballooning $(n=3)$, and laser therapy $(n=3)$ were undergone at previous hospital. In patients with cuff or tip type, Montgomery T-tube $(n=6)$, surgical correction $(n=5)$, metallic stent $(n=3)$, ballooning $(n=2)$, and laser therapy $(n=2)$ were undergone at previous hospital. Patients could undergo more than one procedure at previous hospital. IQR, interquartile range.

type stenosis than in those with subglottic or stoma type stenosis (20.7 vs. $\left.21.7 \mathrm{~kg} / \mathrm{m}^{2}, \mathrm{P}=0.042\right)$. Most patients (72.7\%) underwent tracheostomy due to medical problems such as neurologic disease $(35.3 \%)$ and respiratory failure
(19.2\%). Of the total 99 patients, 26 (26.3\%) were referred to our hospital after failed surgical correction $(n=9)$ and/ or interventional bronchoscopy with stent insertion [Montgomery T-tube $(\mathrm{n}=11)$ or metal stents $(\mathrm{n}=3)$ ], balloon 
Table 2 Treatment modalities

\begin{tabular}{|c|c|c|c|c|}
\hline Variables & Total $(\mathrm{n}=99)$ & Subglottic or stoma type $(n=59)$ & Cuff or tip type $(n=40)$ & $P$ value \\
\hline From injury to detection & $4.0(2.0-7.8)$ & $3.8(1.7-7.0)$ & $4.1(2.3-15.9)$ & 0.160 \\
\hline From detection to intervention & $0.9(0.3-3.0)$ & $0.8(0.3-2.9)$ & $1.1(0.3-3.3)$ & 0.841 \\
\hline \multicolumn{5}{|l|}{ Treatment modalities* } \\
\hline Natural or DUMON stent & $50(50.5)$ & $35(59.3)$ & $15(37.5)$ & 0.041 \\
\hline Montgomery T-tube & $17(17.2)$ & $10(16.9)$ & 7 (17.5) & 1.000 \\
\hline Laser & $20(20.2)$ & $11(18.6)$ & $9(22.5)$ & 0.799 \\
\hline Ballooning & $9(9.1)$ & $5(8.5)$ & $4(10.0)$ & 1.000 \\
\hline
\end{tabular}

Data are presented as $\mathrm{n}(\%)$ or median (IQR). *, patients could undergo more than one procedure. IQR, interquartile range.

dilation ( $\mathrm{n}=5)$, and/or laser cauterization ( $\mathrm{n}=5)$.

Characteristics of the stenosis site were similar between groups (Table 1 and Table S1). Most patients (84.8\%) had tracheal stenosis of grade III or IV. The median length of stenosis was $25 \mathrm{~mm}$ (IQR, 19-35 mm). Although the most common type of stenosis was fibrosis $(74.7 \%)$, mixed stenosis was also common (48.5\%).

\section{Treatment modalities}

Among 99 patients with PTTS, the median time interval from tracheostomy injury to stenosis detection was 4.0 months and from stenosis detection to bronchoscopic intervention was 0.9 months (Table 2 and Table S2). Bronchoscopic interventions were usually performed using a combination of each treatment modality, including stent insertion, laser cauterization, and ballooning. Silicone stent insertion was required more often in patients with subglottic or stoma type stenosis $(45 / 59,76.3 \%)$ than in those with cuff or tip type stenosis $(22 / 40,55.0 \%$; $\mathrm{P}=0.031)$. The frequency of use of Montgomery T-tubes did not differ between groups, but Natural or DUMON stents were more frequently needed in patients with subglottic or stoma type stenosis $(35 / 59,59.3 \%)$ than those with cuff or tip type stenosis $(15 / 40,37.5 \%$; $\mathrm{P}=0.041)$. Each patient underwent a median of two procedures during their treatment course.

\section{Complications and clinical outcomes}

The clinical courses of patients with PTTS are shown in
Figure 2, and the clinical outcomes are shown in Table 3 and Table S3. Acute complications associated with procedures occurred in some patients, including vocal cord dysfunction $(\mathrm{n}=3)$, respiratory distress $(\mathrm{n}=3)$, and excessive bleeding $(\mathrm{n}=2)$. Although chronic complications including granulation tissue overgrowth (43.4\%), mucostasis (41.4\%), stent migration $(33.3 \%)$, and restenosis $(23.5 \%)$ were common, most cases with complications were manageable after additional procedures. There were no significant differences in the complication rates between the two groups. Of the total 99 patients with PTTS, only 20 (20.2\%) were able to perform the PFT within 1 month before and after their first procedures. After intervention, $\mathrm{FEV}_{1}$ was significantly increased in patients with PTTS (median, $1.55-1.72 \mathrm{~L} ; \mathrm{P}=0.035)$.

The median duration of follow-up after the first interventional bronchoscopy was 21.0 months (9.0-45.0 months). Finally, permanent tracheostomy was more frequently needed in patients with cuff or tip type stenosis than those with subglottic or stoma type stenosis ( $50.0 \%$ vs. $18.6 \%, \mathrm{P}=0.002$ ). However, there were no differences in surgical management and mortality rates between the two groups. During the clinical course, only 6 (10.2\%) patients with subglottic or stoma type stenosis and $4(10.0 \%)$ patients with cuff or tip type stenosis were able to maintain airway patency with bronchoscopic intervention without stenting. Successful removal of the tracheostomy tube was significantly more frequent in patients with subglottic or stoma type stenosis than those with cuff or tip type stenosis $(71.2 \%$ vs. $45.0 \%, \mathrm{P}=0.012)$. In addition, 

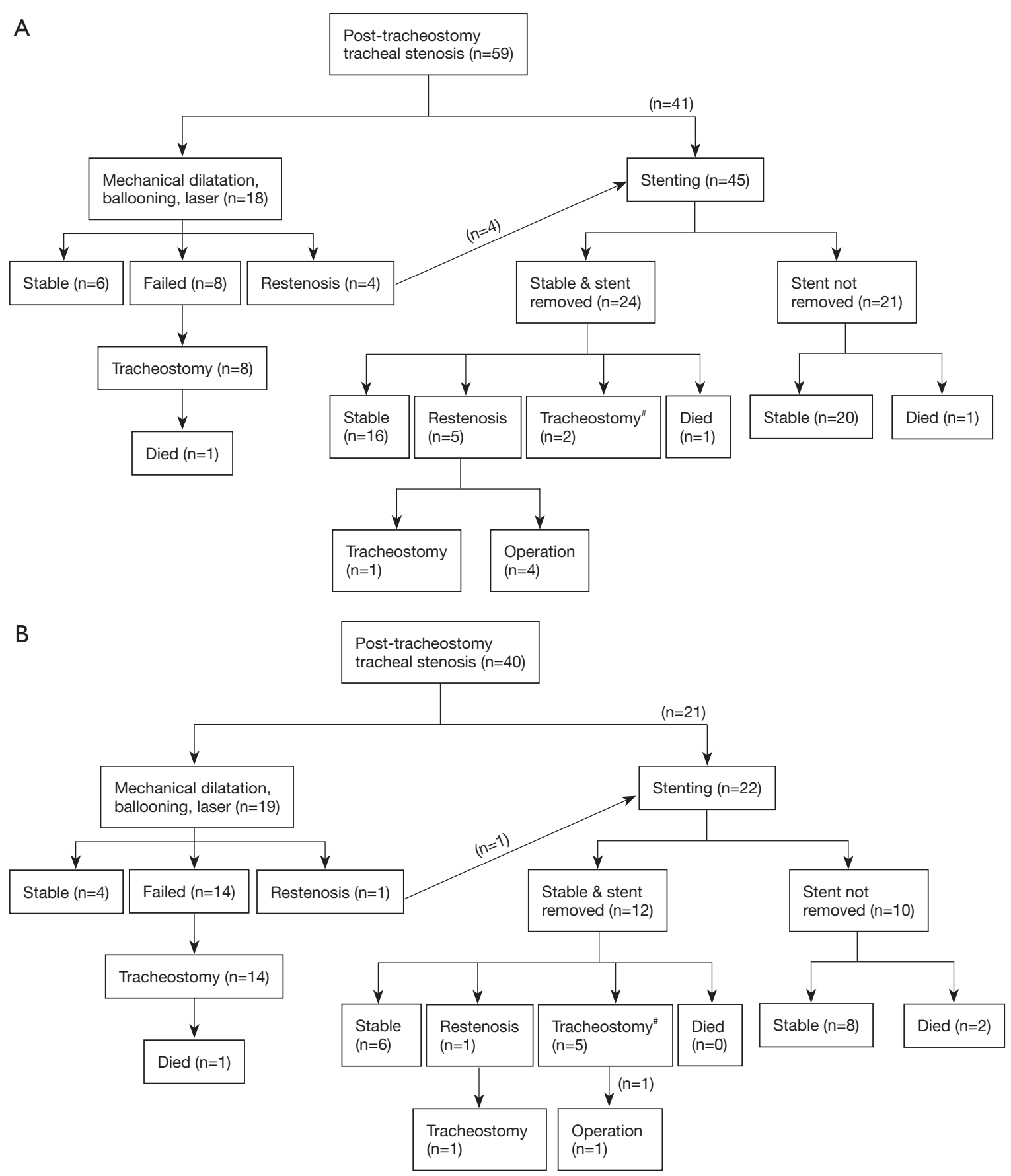

Figure 2 Clinical course of patients with PTTS. (A) Patients with subglottic or stoma type stenosis; (B) patients with cuff or tip type stenosis. \#, these patients underwent tracheostomy because of poor expectoration and/or underlying neurological problems. PTTS, post-tracheostomy tracheal stenosis. 
Table 3 Complications and clinical outcomes

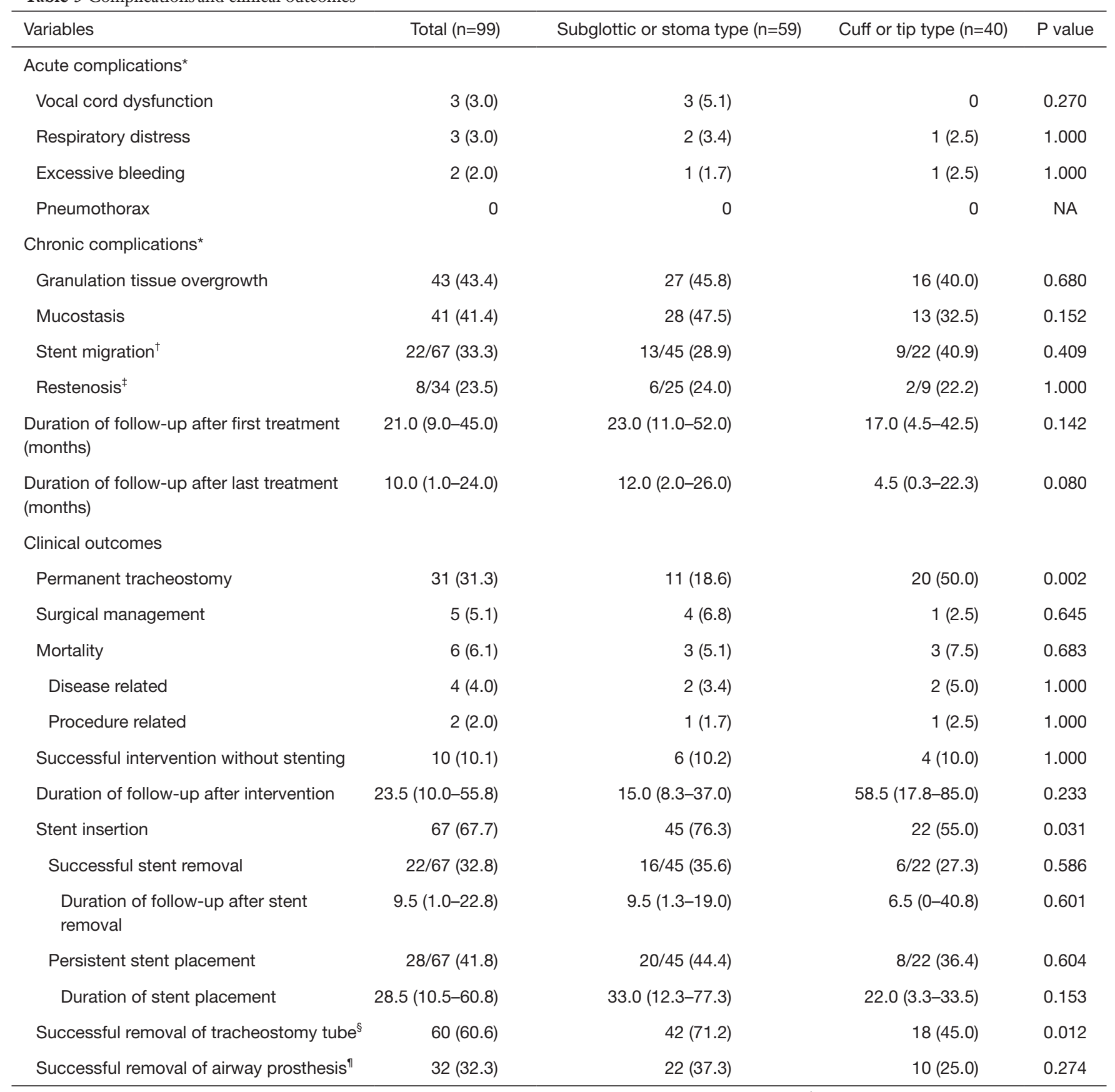

Data are presented as $\mathrm{n}(\%)$ or median (IQR). ${ }^{*}$, patients could have more than one complication; ${ }^{\dagger}$, patients who had a stent inserted were used as the denominator; ${ }^{\ddagger}$, patients who have ever been removed stent were denominator; ${ }^{\S}$, patients who survived and maintained airway patency without a tracheostomy tube or surgical management; ", patients who survived and maintained airway patency without a tracheostomy tube, silicone stent, or surgical management. NA, not applicable; IQR, interquartile range. 
the successful removal of airway prostheses was greater in patients with subglottic or stoma type stenosis than those with cuff or tip type stenosis $(37.3 \%$ vs. $25.0 \%, \mathrm{P}=0.274)$. However, this difference was not statistically significant.

\section{Predictors related to clinical success}

Table 4 shows relationships between patient characteristics and clinical success. Successful removal of the tracheostomy tube was associated with the severity of tracheal stenosis and PTTS type. It was more difficult to remove the tracheostomy tube in patients with stenosis grade IV than those with stenosis grades I-III [adjusted odds ratio (aOR), $0.114 ; 95 \%$ confidence interval $(\mathrm{CI}), 0.021-0.627 ; \mathrm{P}=0.013]$. It was more difficult to remove the tracheostomy tube in patients with cuff or tip type stenosis than those with subglottic or stoma type stenosis (aOR, 0.290; 95\% CI, $0.087-0.967 ; \mathrm{P}=0.044)$. Meanwhile, successful removal of the airway prosthesis was associated with performance status and severity of tracheal stenosis. It was harder to remove the airway prosthesis in patients with poor performance status than in those with good performance status $(\mathrm{aOR}$, $0.226 ; 95 \% \mathrm{CI}, 0.067-0.763 ; \mathrm{P}=0.017)$. It was harder to remove the airway prosthesis in patients with stenosis grade IV than those with stenosis grade I-III (aOR, 0.189; 95\% CI, 0.052-0.681; $\mathrm{P}=0.011$ ).

\section{Discussion}

To the best of our knowledge, this study is the largest to report the results of interventional bronchoscopy in patients with PTTS and the first to compare types of PTTS. There were no differences between types of PTTS in terms of patient characteristics and features of tracheal stenosis, except for BMI. However, patients with subglottic or stoma type stenosis more frequently required silicone stents and less frequently required permanent tracheostomy to maintain airway patency than those with cuff or tip type stenosis. Finally, patients with subglottic or stoma type stenosis showed more favorable outcomes, measured by the successful removal of the tracheostomy tube and closure of the stoma without surgical intervention or procedureand disease-related death, than those with cuff or tip type stenosis.

PTTS can be categorized according to the stenosis site and pathophysiology such as subglottic, stoma, cuff, and tip granuloma type (9). Subglottic type can be caused by incorrect needle puncture or incision during the tracheostomy procedure. According to an observation study, PTTS of subglottic type more frequently occurred in patients with percutaneous dilational tracheostomy (PDT) than those with surgical tracheostomy (SGT) [25/105 (23.8\%) vs. 3/41 (7.3\%), $\mathrm{P}=0.033]$ (28). Stoma type is associated with inadequate tracheal incision and ongoing stomal infection. These types of damage during tracheostomy procedures may result in abnormal wound healing and excess granulation tissue formation around damaged cartilage (29). In a previous study, stenosis of the stoma site was the most common type in patients with PTTS (30). Cuff type stenosis is caused by ischemic mucosal damage when cuff-to-tracheal wall tension exceeds the mucosa capillary perfusion pressure, usually by 20 to $30 \mathrm{mmHg}$ (31). Risk can be minimized by the use of largevolume and low-pressure cuffs (32). Lastly, tip granuloma type stenosis results from the inappropriate positioning of the tube or excessive recurrent suction (9). Although we have no data regarding detailed tracheostomy procedures and cuff types, subglottic or stoma type PTTS may be more frequently encountered in clinical settings than cuff or tip type stenosis, because PDT and high-volume low-pressure cuffs are more widely used than SGT and low-volume highpressure cuffs $(28,32,33)$.

Few studies have evaluated the incidence, treatment modalities, and prognosis of each type of PTTS. The present study categorized PTTS into two groups according to similar mechanisms of tracheal stenosis, and then assessed differences in the demographic data, treatment modalities, and clinical outcomes between the two groups. Although there were no differences in baseline characteristics, successful removal of the tracheostomy tube was achieved more frequently in patients with subglottic or stoma type stenosis than those with cuff or tip type stenosis. This finding was supported in a multiple logistic regression model. In addition, classification of PTTS type and stenosis grade were important factors predicting the successful removal of the tracheostomy tube, and performance status was critical for the successful removal of airway prostheses including the tracheostomy tube, Montgomery T-tube, and airway silicone stents.

In present study, stent migration is a common complication. Stent can be migrated more frequently when stent is placed closer to the subglottic area, because movement of the head and neck or coughing tend to induce stent migration $(34,35)$. However, present study did not show an increased incidence of stent migration across each type of PTTS from tip type to subglottic type (Table S3), because we had performed the external fixation with a nylon 


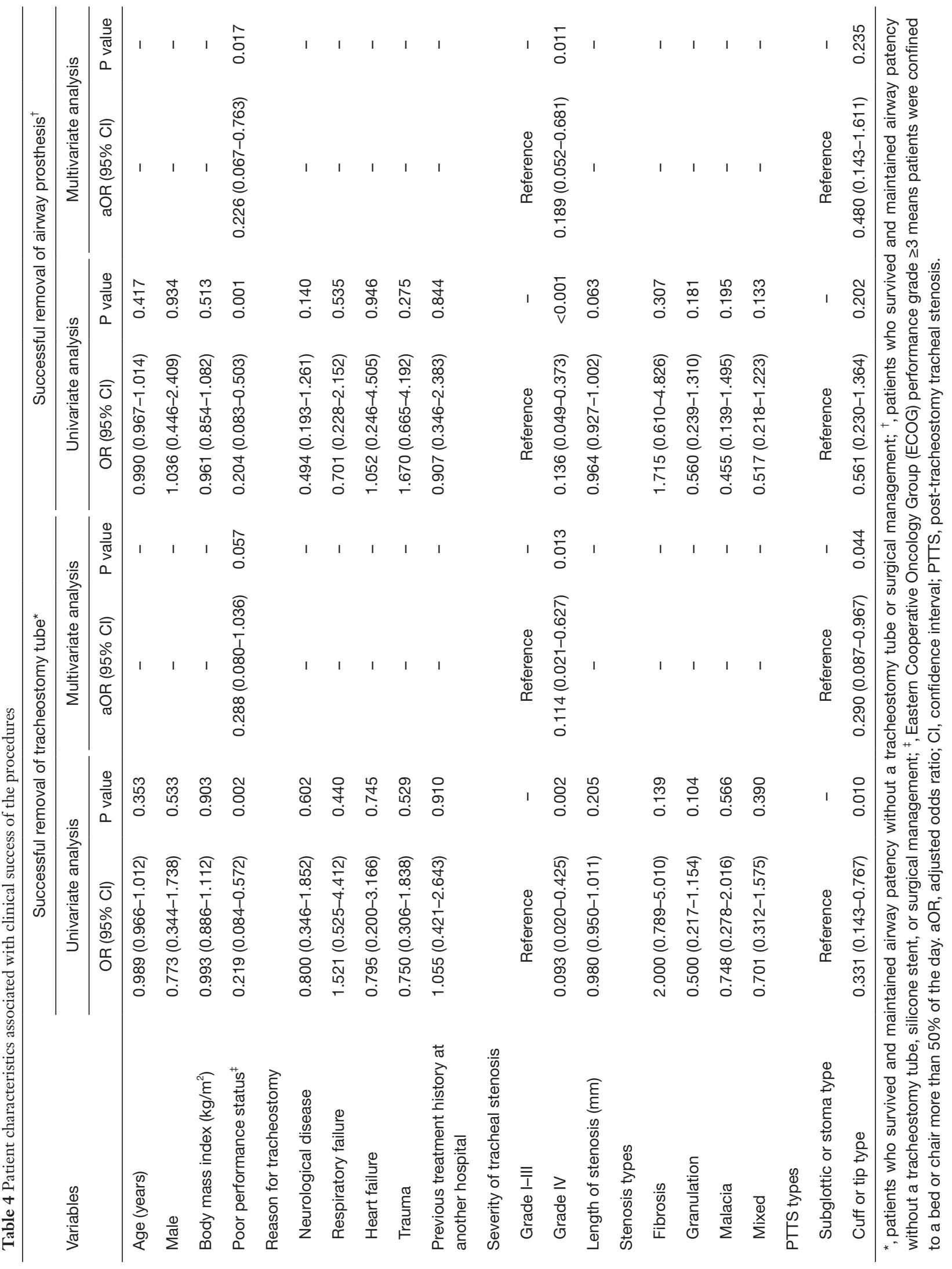


thread in the majority of patients with tracheal stenosis. In these 22 patients with stent migration, 32 events of migration were occurred during 53.8 years of the whole period of stent insertion. Arithmetically, migration event was occurred every 1.9 years. Almost cases of migration were occurred after spontaneous cut of thread.

There are several limitations to the present study that should be acknowledged. First, our study was retrospective and conducted at a single institution. In addition, our institution is almost the only hospital to actively perform the rigid bronchoscopy and insertion of silicone stent in Korea. Actually, 26 patients were transferred to our hospital after some treatment for PTTS, 72 patients were transferred to our hospital after confirmation of PTTS without any treatment for PTTS, and only one patient was diagnosed with PTTS in our hospital. There is therefore a possibility that selection bias was present. Second, although the present study is the biggest study of PTTS ever conducted, the number of patients was too small to compare characteristics and outcomes among all four types of PTTS. We therefore only discovered differences between "subglottic or stoma type" stenosis and "cuff or tip type" stenosis. Therefore, further studies are needed to evaluate the characteristics of each type. Third, we failed to obtain detailed information on tracheostomy procedure such as PDT, SGT, and cuff types because of difficulties in obtaining complete medical records. Further studies are needed to characterize stenosis type according to detailed tracheostomy procedures and tracheostomy maintenance methods.

\section{Conclusions}

In conclusion, there were no significant differences in baseline characteristics between patients with two different types of PTTS. However, patients with subglottic or stoma type stenosis had more favorable outcomes than those with cuff or tip type stenosis. Therefore, distinguishing between types of PTTS could be important in predicting patient prognosis.

\section{Acknowledgements}

None.

\section{Footnote}

Conflicts of Interests: The authors have no conflicts of interests to declare.
Ethical Statement: This retrospective observational study received Institutional Review Board of Samsung Medical Center approval (IRB No. 2016-08-139). The need for informed consent was waived because patient information was anonymized and de-identified prior to analysis.

\section{References}

1. Sarper A, Ayten A, Eser I, et al. Tracheal stenosis aftertracheostomy or intubation: review with special regard to cause and management. Tex Heart Inst J 2005;32:154-8.

2. Streitz JM Jr, Shapshay SM. Airway injury after tracheotomy and endotracheal intubation. Surg Clin North Am 1991;71:1211-30.

3. De Leyn P, Bedert L, Delcroix M, et al. Tracheotomy: clinical review and guidelines. Eur J Cardiothorac Surg 2007;32:412-21.

4. Norwood S, Vallina VL, Short K, et al. Incidence of tracheal stenosis and other late complications after percutaneous tracheostomy. Ann Surg 2000;232:233-41.

5. Bisson A, Bonnette P, el Kadi NB, et al. Tracheal sleeve resection for iatrogenic stenoses (subglottic laryngeal and tracheal). J Thorac Cardiovasc Surg 1992;104:882-7.

6. Rea F, Callegaro D, Loy M, et al. Benign tracheal and laryngotracheal stenosis: surgical treatment and results. Eur J Cardiothorac Surg 2002;22:352-6.

7. Colt HG, Harrell JH. Therapeutic rigid bronchoscopy allows level of care changes in patients with acute respiratory failure from central airways obstruction. Chest 1997;112:202-6.

8. Galluccio G, Lucantoni G, Battistoni P, et al. Interventional endoscopy in the management of benign tracheal stenoses: definitive treatment at long-term follow-up. Eur J Cardiothorac Surg 2009;35:429-33; discussion 933-4.

9. Epstein SK. Late complications of tracheostomy. Respir Care 2005;50:542-9.

10. Shin B, Kim K, Jeong BH, et al. Clinical significance of differentiating post-intubation and post-tracheostomy tracheal stenosis. Respirology 2017;22:513-20.

11. Brouns M, Jayaraju ST, Lacor C, et al. Tracheal stenosis: a flow dynamics study. J Appl Physiol (1985) 2007;102:1178-84.

12. Stoelben E, Koryllos A, Beckers F, et al. Benign stenosis of the trachea. Thorac Surg Clin 2014;24:59-65.

13. Jeong BH, Um SW, Suh GY, et al. Results of interventional bronchoscopy in the management of postoperative tracheobronchial stenosis. J Thorac 
Cardiovasc Surg 2012;144:217-22.

14. Colt HG, Dumon JF. Airway stents. Present and future. Clin Chest Med 1995;16:465-78.

15. Kim H. Stenting therapy for stenosing airway disease. Respirology 1998;3:221-8.

16. Park HY, Kim H, Koh WJ, et al. Natural stent in the management of post-intubation tracheal stenosis. Respirology 2009;14:583-8.

17. Brichet A, Verkindre C, Dupont J, et al. Multidisciplinary approach to management of postintubation tracheal stenoses. Eur Respir J 1999;13:888-93.

18. Lim SY, Kim H, Jeon K, et al. Prognostic factors for endotracheal silicone stenting in the management of inoperable post-intubation tracheal stenosis. Yonsei Med J 2012;53:565-70.

19. Dumon JF. A dedicated tracheobronchial stent. Chest 1990; 97:328-32.

20. Noppen M, Schlesser M, Meysman M, et al. Bronchoscopic balloon dilatation in the combined management of postintubation stenosis of the trachea in adults. Chest 1997;112:1136-40.

21. Verma A, Park HY, Lim SY, et al. Posttuberculosis tracheobronchial stenosis: use of CT to optimize the time of silicone stent removal. Radiology 2012;263:562-8.

22. Ryu YJ, Kim H, Yu CM, et al. Comparison of natural and Dumon airway stents for the management of benign tracheobronchial stenoses. Respirology 2006;11:748-54.

23. Kim HJ, Koh WJ, Suh GY, et al. The Usefulness and Safety of Natural Stent in a Canine Model of Tracheal Stenosis. Tuberc Respir Dis 2002;53:431-8.

24. Wahidi MM, Ernst A. The Montgomery T-tube tracheal stent. Clin Chest Med 2003;24:437-43.

25. Carretta A, Casiraghi M, Melloni G, et al. Montgomery T-tube placement in the treatment of benign tracheal lesions. Eur J Cardiothorac Surg 2009;36:352-6; discussion 356.

26. Oken MM, Creech RH, Tormey DC, et al. Toxicity and response criteria of the Eastern Cooperative Oncology Group. Am J Clin Oncol 1982;5:649-55.

27. Myer CM 3rd, O'Connor DM, Cotton RT. Proposed grading system for subglottic stenosis based on endotracheal tube sizes. Ann Otol Rhinol Laryngol 1994;103:319-23.

28. Koitschev A, Simon C, Blumenstock G, et al. Suprastomal tracheal stenosis after dilational and surgical tracheostomy in critically ill patients. Anaesthesia 2006;61:832-7.

29. Pearson FG, Andrews MJ. Detection and management of tracheal stenosis following cuffed tube tracheostomy. Ann Thorac Surg 1971;12:359-74.

30. Zias N, Chroneou A, Tabba MK, et al. Post tracheostomy and post intubation tracheal stenosis: report of 31 cases and review of the literature. BMC Pulm Med 2008;8:18.

31. Wood DE, Mathisen DJ. Late complications of tracheotomy. Clin Chest Med 1991;12:597-609.

32. Grillo HC, Cooper JD, Geffin B, et al. A low-pressure cuff for tracheostomy tubes to minimize tracheal injury. A comparative clinical trial. J Thorac Cardiovasc Surg 1971;62:898-907.

33. Raghuraman G, Rajan S, Marzouk JK, et al. Is tracheal stenosis caused by percutaneous tracheostomy different from that by surgical tracheostomy? Chest 2005;127:879-85.

34. Colt HG, Harrell J, Neuman TR, et al. External fixation of subglottic tracheal stents. Chest 1994;105:1653-7.

35. Miwa K, Takamori S, Hayashi A, et al. Fixation of silicone stents in the subglottic trachea: preventing stent migration using a fixation apparatus. Ann Thorac Surg 2004;78:2188-90.
Cite this article as: Shin B, Kim K, Jeong BH, Eom JS, Song WJ, Kim H. Clinical implications of differentiating between types of post-tracheostomy tracheal stenosis. J Thorac Dis 2017;9(11):4413-4423. doi: 10.21037/jtd.2017.10.99 


\section{Supplementary}

Table S1 Baseline characteristics according to each type

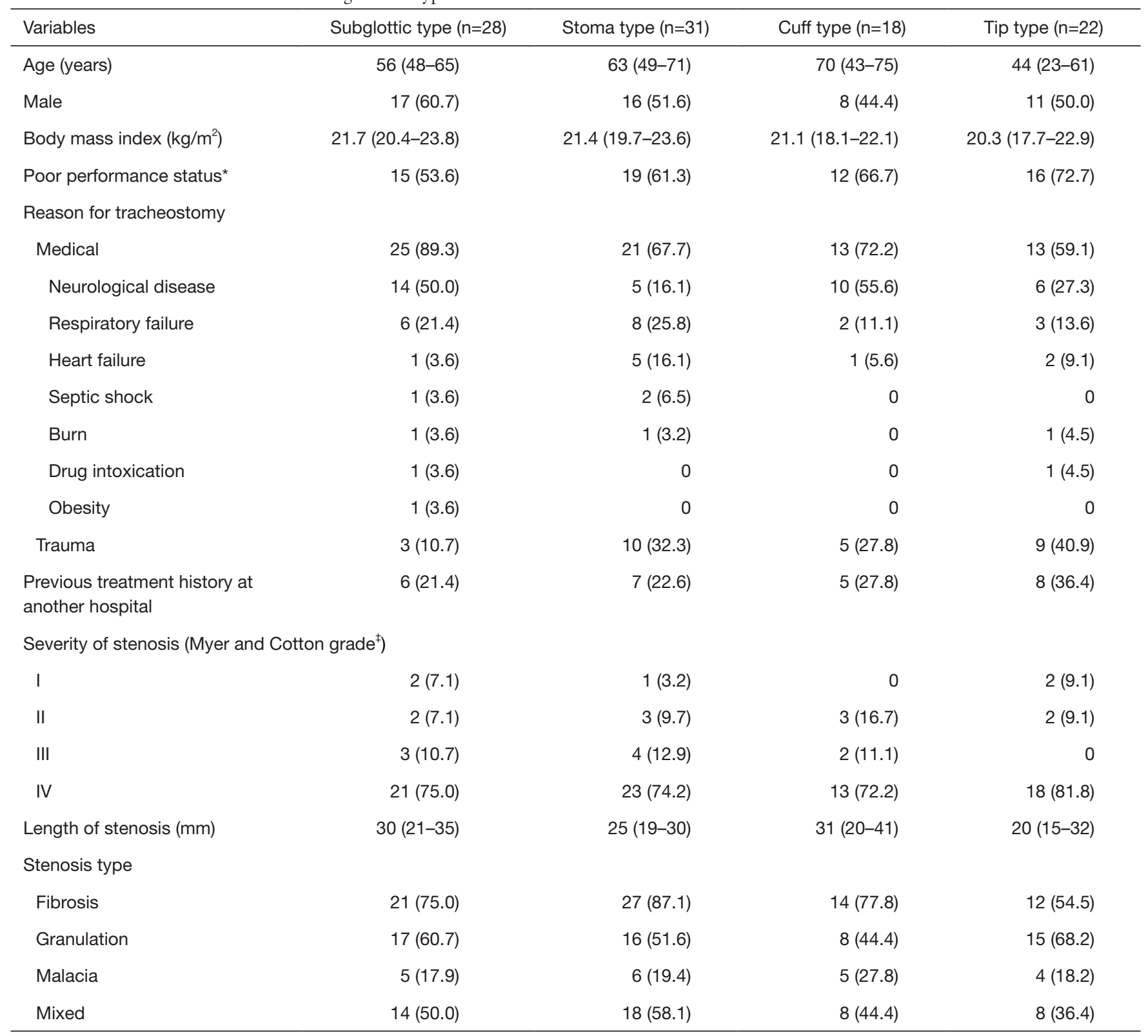

Data are presented as $n(\%)$ or median (IQR). *, Eastern Cooperative Oncology Group (ECOG) performance grade $\geq 3$ means patients were confined to a bed or chair more than $50 \%$ of the day; ${ }^{\ddagger}$, categorization based on the percentage of reduction in cross-sectional area: grade 1, $\leq 50 \%$ lumenal stenosis; grade II, 51-70\% lumenal stenosis; grade III, 71-99\% lumenal stenosis; grade IV, no lumen, intubation or tracheostomy state due to respiratory failure before interventional bronchoscopy. IQR, interquartile range. 
Table S2 Treatment modalities according to each type

\begin{tabular}{lcccc}
\hline Variables & Subglottic type $(\mathrm{n}=28)$ & Stoma type $(\mathrm{n}=31)$ & Cuff type $(\mathrm{n}=18)$ & Tip type $(\mathrm{n}=22)$ \\
\hline Time interval (months) & & & & \\
$\quad$ From injury to detection & $4.0(2.0-9.9)$ & $3.1(1.7-6.9)$ & $3.5(1.8-11.7)$ & $5.3(2.7-18.4)$ \\
$\quad$ From detection to intervention & $0.7(0.2-5.5)$ & $0.9(0.3-2.4)$ & $0.9(0.3-4.9)$ & $1.1(0.4-2.7)$ \\
Treatment modalities & & & & \\
Silicone stent & $23(82.1)$ & $22(71.0)$ & $10(55.6)$ & $12(54.5)$ \\
$\quad$ Natural or DUMON stent & $18(64.3)$ & $17(54.8)$ & $6(33.3)$ & $9(40.9)$ \\
$\quad$ Montgomery T-tube & $5(17.9)$ & $5(16.1)$ & $4(22.2)$ & $3(13.6)$ \\
Laser & $8(28.6)$ & $3(9.7)$ & $7(38.9)$ & $2(9.1)$ \\
Ballooning & $1(3.6)$ & $4(12.9)$ & $2(11.1)$ & $2(1-4)$ \\
Number of interventional bronchoscopies & $2(1-3)$ & $2(1-3)$ & $2(1-3)$ \\
\hline
\end{tabular}

Data are presented as $\mathrm{n}(\%)$ or median (IQR). ${ }^{*}$, patients could undergo more than one procedure. IQR, interquartile range.

Table S3 Complications and clinical outcomes according to each type

\begin{tabular}{|c|c|c|c|c|}
\hline Variables & Subglottic type $(n=28)$ & Stoma type $(n=31)$ & Cuff type $(n=18)$ & Tip type $(n=22)$ \\
\hline \multicolumn{5}{|l|}{ Acute complications* } \\
\hline Vocal cord dysfunction & $3(10.7)$ & 0 & 0 & 0 \\
\hline Respiratory distress & $1(3.6)$ & $1(3.2)$ & $1(5.6)$ & 0 \\
\hline Excessive bleeding & 0 & $1(3.2)$ & $1(5.6)$ & 0 \\
\hline Pneumothorax & 0 & 0 & 0 & 0 \\
\hline \multicolumn{5}{|l|}{ Chronic complications ${ }^{*}$} \\
\hline Granulation tissue overgrowth & $15(53.6)$ & $12(38.7)$ & $7(38.9)$ & $9(40.9)$ \\
\hline Mucostasis & $15(53.6)$ & $13(41.9)$ & $6(33.3)$ & $7(31.8)$ \\
\hline Stent migration $^{\dagger}$ & 9/23 (39.1) & 4/22 (18.2) & $5 / 10(50.0)$ & 4/12 (33.3) \\
\hline Restenosis $^{\ddagger}$ & $3 / 12(25.0)$ & $3 / 13(23.1)$ & $1 / 5(20.0)$ & $1 / 4(25.0)$ \\
\hline Duration of follow-up after first treatment (months) & $29(11-60)$ & $19(10-45)$ & $14(4-33)$ & $21(8-49)$ \\
\hline Duration of follow-up after last treatment (months) & $14(2-34)$ & $10(2-22)$ & $5(1-17)$ & $4(0-27)$ \\
\hline \multicolumn{5}{|l|}{ Clinical outcomes } \\
\hline Permanent tracheostomy & $3(10.7)$ & $8(25.8)$ & $9(50.0)$ & $11(50.0)$ \\
\hline Surgical management & $2(7.1)$ & $2(6.5)$ & $1(5.6)$ & 0 \\
\hline Mortality & $1(3.6)$ & $2(6.5)$ & $2(11.1)$ & $1(4.5)$ \\
\hline Disease related & 0 & $2(6.5)$ & $2(11.1)$ & 0 \\
\hline Procedure related & $1(3.6)$ & 0 & 0 & $1(4.5)$ \\
\hline Successful intervention without stenting & $3(10.7)$ & $3(9.7)$ & $2(11.1)$ & $2(9.1)$ \\
\hline Stent insertion & $23(82.1)$ & $22(71.0)$ & $10(55.6)$ & $12(54.5)$ \\
\hline Successful stent removal & 7/23 (30.4) & 9/22 (40.9) & $3 / 10(30.0)$ & $3 / 12(25.0)$ \\
\hline Persistent stent placement & $12 / 23(52.2)$ & $8 / 22(36.4)$ & $3 / 10(30.0)$ & $5 / 12(41.7)$ \\
\hline Successful removal of tracheostomy tube $e^{\S}$ & $22(78.6)$ & $20(64.5)$ & $8(44.4)$ & $10(45.5)$ \\
\hline Successful removal of airway prosthesis" & $10(35.7)$ & $12(38.7)$ & $5(27.8)$ & $5(22.7)$ \\
\hline
\end{tabular}

Data are presented as $\mathrm{n}(\%)$ or median (IQR). ${ }^{*}$, patients could have more than one complication; ${ }^{\dagger}$, patients who had a stent inserted were used as the denominator; ${ }^{\ddagger}$, patients who have ever been removed stent were denominator; ${ }^{\S}$, patients who survived and maintained airway patency without a tracheostomy tube or surgical management; ", patients who survived and maintained airway patency without a tracheostomy tube, silicone stent, or surgical management. IQR, interquartile range. 